\title{
La física de la sensación aristotélica de acuerdo a Teofrasto (Física $V)^{1}$
}

André Laks

Université de Paris-Sorbonne/Universidad Panamericana

laks.andre@gmail.com

Hace unos 15 años tuvo lugar un debate entre los intérpretes anglosajones que pensaban, junto con R. Sorabji, que para Aristóteles el acto perceptivo no se da sin una alteración fisiológica del órgano sensorial (sin significar que se reduzca a ello) ${ }^{2}$, y quienes, como Burnyeat, sostenían - por el contrario-que al no suponer ningún «proceso material», la teoría aristotélica de la percepción compete a «una física de las formas puras» (Burnyeat 1996; 163). La primera interpretación se apoya sobre una serie de afirmaciones en el tratado Acer-

\footnotetext{
${ }^{1}$ Desde la publicación original de este artículo [en M. Canto y P. Pellegrin (eds.), Le Style de la pensée. Recueil d'hommages à Jacques Brunschwig, Paris, 2002, pp. 353-374], el debate al que hago referencia al inicio del texto no ha dejado de afinarse y ha dado lugar a una larga e impresionante síntesis por parte de V. Caston: «The Spirit and the Letter, Aristotle on Perception» en R. Salles (ed.), Metaphysics, Soul and Ethics in Ancient Thought. Themes from the work of Richard Sorabji, Oxford 2005, pp. 245-320 (con una amplia bibliografía). Dado que en este artículo no tomo postura en dicho debate, sino que sólo dirijo la atención sobre una fuente —unos fragmentos de Teofrasto- que según sé no se ha utilizado en la discusión, no me parece superflua la traducción de esta contribución a otro idioma, aunque la información difícilmente llegue mejor al público anglosajón por medio del castellano que por medio del francés. Agradezco mucho a René Ceceña por su traducción y a Alberto Ross por invitarme a dar esta ponencia en la Universidad Panamericana. 2 «Cuando veo una escena en colores, la substancia gelatinosa de mi ojo se tiñe de las manchas cuyo color, formas y posiciones corresponden a esta escena» (Sorabji 1991: 228; Sorabji 1992: 209ss y Sorabji 2001).
} 
ca del alma de Aristóteles, de acuerdo a las cuales el órgano sensorial recibe las formas sensibles y es afectado por ellas, e incluso que el órgano de los sentidos en primera instancia es diferente al objeto sensible, deviene como él y es potencialmente lo que este último es en acto. Esta lectura podemos calificarla como literal'; la segunda interpretación contesta invocando la elasticidad del vocabulario aristotélico: según Burnyeat, lo que se transmite de lo sensible al sentido no es un movimiento sino un «cuasi-movimiento», el ojo que percibe no cambia, sino sólo «cuasi-cambia», y la afectación sensorial en consecuencia no es sino una cuasi-afectación. Esto es lo que escribe Burnyeat: «Desgraciadamente, para los partidarios de los procesos materiales, este movimiento, esta kívๆots tıs, no es una especie de movimiento, sino un cuasi-movimiento, un movimiento de alguna manera» (Burnyeat refiere al empleo llamado alienans del indefinido tıs.). ${ }^{4}$ Sabemos, de hecho, que tratándose de la percepción, Aristóteles recurre al vocabulario de la afección con mucha reserva, y un tanto a disgusto 5 .

En el curso del debate se ha invocado la tradición de los comentadores de Aristóteles, y Burnyeat ha podido apelar a ella, puesto que, en términos generales, los comentadores coinciden con el espíritu de su interpretación (ver Burnyeat 1996, 150, n. 1; 160ss). Es comprensible que Sorabji, inversamente, vea en ello el efecto de una distorsión progresiva, de la cual ha distinguido las etapas retrazando la historia de lo que

\footnotetext{
${ }^{3}$ Sorabji 1992; 213 (escribiendo a propósito de 429a15ss): «In other words, it involves the literal coloration of the organ of sight».

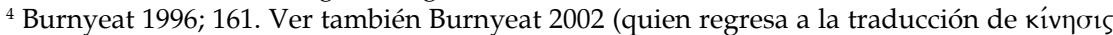
por «movimiento», demasiado restrictivo pues no corresponde sino a una de las especies aristotélicas del cambio, el cambio local, e inadaptado a un contexto donde el término se refiere la mayor de las veces a un cambio cualitativo (o cuasi-cualitativo), que Aristóteles

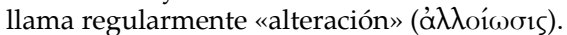

${ }^{5}$ Ver especialmente Acerca del alma, II, 5, 417b2-5, b12-16, 417b22-418a3.
} 
él llama la «desmaterialización» de la explicación aristotélica; una desmaterialización que culminaría con la interpretación que Brentano, apoyándose en Tomás de Aquino, debía dar de la teoría aristotélica de la percepción en términos de pura intencionalidad (ver Sorabji 1991, 228; y Sorabji 1992; 225). Los comentadores mencionados por Burnyeat son, recorriéndolos en sentido cronológicamente inverso a partir de Tomás de Aquino: Alberto Magno, Avicena, Filopón, Temistio y Alejandro de Afrodisia ${ }^{6}$. Ahora bien, se puede remontar aun más lejos, hasta Teofrasto, quien es, curiosamente, el gran ausente en este debate. Lo que me propongo aquí es únicamente presentar el material que a este respecto está a nuestra disposición.

Teofrasto escribió, entre muchas otras obras, una Física (Фuбıká) en ocho libros. Como suele ocurrir, está perdida, y sólo la conocemos a través de unos pocos fragmentos y reportes $^{7}$. Temistio, quien hace uso de ella en su comentario al De anima de Aristóteles, nos indica que los libros 4 y 5 de esta obra constituyen un Tratado del alma; un tratado que puede originalmente haber sido independiente, pero cuya inclusión en una Física es enteramente conforme al programa de Aristóteles (ver Acerca del alma, I, 1, 402a4-6). La descripción que Temistio nos da de la obra corresponde bien a las características de otra obra de Teofrasto que tenemos la suerte de co-

\footnotetext{
${ }^{6}$ Sobre Tomás, ver Burnyeat 2001.

${ }^{7}$ Diógenes Laercio, v, 46, menciona muchas obras de Física: «Acerca de la naturaleza» (Пєpi

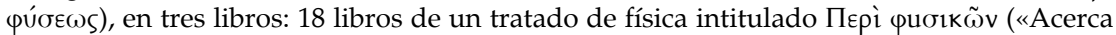
de las cuestiones naturales»); y el que podría ser un compendio de dos libros de una de

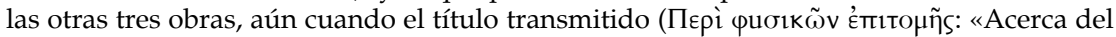
compendio del tratado de física») no esté exento de dificultades: uno esperaría el nomina-

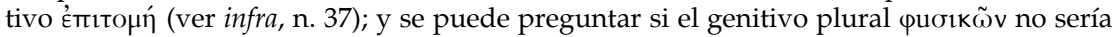
un masculino más que un neutro (se trataría entonces de un «compendio de físicos»). Otros títulos son constatados en la tradición (Historia física, Historia natural, etcétera). Es comprensible que las hipótesis sobre la relación entre los diferentes títulos y el contenido de las obras correspondientes sean numerosas. Ver Steinmetz 1964: 2 y 349ss; y Gottschalk 1967.
} 
nocer, la así llamada Metafísica. Dice Temistio en su comentario del De Anima III, 5 con relación a los libros de Teofrasto acerca del alma:

T1 = Temistio, en De anima. III, 5, p 108 8-11 (307 , 17-21 FHS\&G) Resultaría largo citar los planteamientos que siguen, aun cuando su exposición no sea larga, sino al más alto grado ceñida y concisa, por lo menos en lo que respecta a la expresión; pues por lo que respecta a los contenidos, están llenos de numerosas aporías, de numerosas pausas, y de numerosas soluciones. Y se encuentran en el quinto libro de la Física, que es el segundo libro del Tratado del alma ${ }^{8}$.

Además de Temistio, la principal fuente de los fragmentos conservados de esa parte de la Física de Teofrasto es el filósofo neo-platónico Prisciano de Lidia quien, en un comentario conocido con el nombre de «metáfrasis», utiliza la obra para desarrollar una teoría neoplatónica de la sensación9. Los fragmentos versan sobre tres series de cuestiones correspondientes a la segunda parte del De anima de Aristóteles (II, 5-III, 13): la teoría de la sensación, la teoría de la imaginación y la teoría del intelecto. Todas poseen un marcado carácter exegético. Desde ese punto de vista, se les puede vincular con los fragmentos del primer libro de este mismo tratado, que se presenta, como tuve la oportunidad de sostener en otro lugar, en forma de comentario al comienzo de la Física

\footnotetext{
${ }^{8}$ El texto griego de los pasajes traducidos figura en el apéndice. La descripción se aplica también al opúsculo metafísico (ver Laks y Most 1993: XIX, n. 2). Ver igualmente Prisciano, Metaphrasis in Theophrastum, p. 36, 6-9 Bywater.

${ }_{9}^{9}$ Prisiciano conocía bien a Teofrasto (ver Steinmetz 1964: 349), quien remite al prólogo de las Solutiones ad Chosroen, donde Teofrasto es explícitamente citado dentro de los escritos utilizados (42, 3-7 Bywater).
} 
de Aristóteles (ver Laks, 1998/2007).

La problematización y la explicación del texto de Aristóteles parecen haber proveído la materia exclusiva del quinto libro, que Temistio menciona en T1. La indicación es confirmada por Prisciano, en una frase que sirve de transición entre la sección de su metáfrasis consagrada a la sensación y la que trata sobre la imaginación:

$\mathrm{T} 2=$ Prisciano, p. 22. 33ss. Bywater

Pasemos a lo siguiente, llevando a su término, a partir de un nuevo inicio, el resto del libro quinto.

El comentario de Teofrasto debe haber sido sensiblemente más corto que el texto de referencia, puesto que el segundo libro del tratado Acerca del alma de Teofrasto (el libro V de su Física) abordaba también temas que figuran en el tercer libro de la obra de Aristóteles. Éste concuerda con la brevedad sobre la que insiste Temistio (T1). Es verosímil que en lo esencial, la primera parte del De anima de Aristóteles (I, 1-II, 5) fuera el objeto del primer libro del Tratado del alma de Teofrasto (el libro IV de su Física $)^{10}$. ¿Es el opúsculo teofrastiano Acerca de las sensaciones, transmitido de manera independiente, un extracto de este libro? El opúsculo está, en efecto, consagrado a las teorías prearistotélicas de la sensación y del pensamiento, a las cuales por su parte Aristóteles remite en los capítulos iniciales del De anima (especialmente I, 2) ${ }^{11}$. Además, la noción

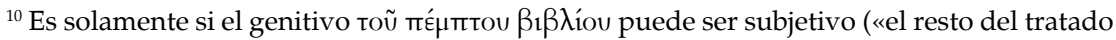
Acerca del alma, en este caso el quinto libro») que se podría pensar que el cuarto libro (que abordaba también otros temas, ver infra, n. 19) incluía el tratamiento de la sensación. Sin embargo, esta construcción es poco natural.

${ }^{11}$ Steinmetz (1964: 338), ya había planteado la cuestión, pero sin continuarla, puesto que descarta deliberadamente $y$, a decir verdad arbitrariamente, el examen de la psicología de la reconstrucción de la Física de Teofrasto (p. 13).
} 
de "asimilación", sobre la cual, como lo veremos, Teofrasto se había ocupado en el libro V de la Física, esclarece también de cierta manera la estructuración doxográfica del opúsculo, el cual no solamente clasifica las diferentes doctrinas en función de los dos principios de lo semejante y lo contrario, sino que otorga también cierta prioridad, a la vez cronológica y lógica, a las teorías que apelan a la similitud (ver Mansfeld 1996; 170ss).

En lo que sigue, sólo examinaré los fragmentos teofrastianos relativos a la doctrina aristotélica de la sensación, para lo cual dependemos casi exclusivamente - con muy pocas excepciones, como la de un pasaje muy interesante de Simplicio-, de Prisciano ${ }^{12}$.

Las «soluciones» formuladas en el quinto libro de la Física de Teofrasto iban indudablemente en el sentido de una justificación de la doctrina y expresión aristotélicas. Teofrasto parece haber procedido mediante la yuxtaposición de unidades en donde la formulación de una dificultad doctrinal era seguida por una proposición de solución al término de una eventual «búsqueda» (zêtêsis) intermedia, caracterizada por una «atención detenida» (epistasis) al texto de Aristóteles, según la expresión de Prisciano (es interesante que Prisciano distinga entre la epistasis y la formulación de una dificultad, aporia) ${ }^{13}$. Estamos entonces frente a una explicación del texto, con sus esclarecimientos y sus complementos, pero donde no parece tratarse sino de volver a decir lo que Aristóteles dijo ${ }^{14}$. Las zonas de correspondencia entre Aristóteles y

\footnotetext{
${ }^{12}$ Otro testimonio aislado, debido a Filopón, no hace sino confirmar a Prisciano (ver infra, n. 29).

${ }^{13}$ Siguiendo la terminología que propuse en la introducción a la edición Laks y Most (1993) del opúsculo metafísico, las aporías son en este caso «catárticas» (ver p. XVIII).

${ }^{14}$ Ver T1, además de Prisciano, p. 14, 23-25 Bywater = 275B FHS\&G: «...este punto que fue juzgado por Teofrasto debe ser el objeto de simple atención, y no fue por su parte objeto
} 
Teofrasto son en todo caso amplias. En particular, Teofrasto acepta que ninguna sensación se efectúa por contacto directo del órgano sensorial con el sensible, y que todas las sensaciones suponen un intermediario, sea éste interno o externo ${ }^{15}$.

¿Se limita Teofrasto a clarificar? Prisciano no es de esta opinión, pues, aun sabiendo que la correspondencia es fundamental, se interesa desde su perspectiva neoplatónica, por lo que Teofrasto añade a la doctrina aristotélica, y por la continuación que da a ciertas dificultades que ésta suscita. Respecto al mecanismo de la vista, indica lo siguiente:

T3 =Prisciano, p. 7, 20-23 (275 A 1-4 FHS\&G)

Pues nuestro objeto no es por ahora proseguir la cuestión con detalle articulando estos puntos, sino reunir los planteamientos de Teofrasto cuando, añadiendo elementos a la tradición de Aristóteles, va más allá, y, cuando propone una tesis en el curso de un desarrollo aporético, conducirla a su término en la medida de lo posible.

La formulación de Prisciano es interesante. En ella se subraya la novedad de ciertas afirmaciones de Teofrasto e implica que algunas de sus propuestas seguirían la dirección de las del propio Prisciano; es decir, básicamente la postura de Jámblico, en quien Prisciano se inspira directamente ${ }^{16}$. De hecho,

de ninguna aporía».

${ }^{15}$ Ver 275A, 5-7 FHS\&G (del cual T3 constituye la continuación): «Con toda evidencia, [Teofrasto] juzga también que ninguna sensación toca lo sensible; pues no es razonable, dice, admitir la ausencia de comunidad y similitud en el seno de lo que es homogéneo» (ver también 275B FHS\&G, así como T9).

${ }^{16}$ Se puede leer antes de T3: «Tal es la manera de proceder en el tratamiento de cada una de las sensaciones, lo cual hay que retomar de las investigaciones realizadas por Jámblico en su escrito Acerca del alma, de donde proviene lo que acabamos de escribir en forma condensada, con la intención de esbozar los contornos del tratamiento exacto de cada una» (7, 16-20). Acerca de la teoría de la sensación en los neoplatónicos tardíos, ver Hadot 1997, 
en numerosas ocasiones a lo largo de su tratado, Prisciano considera que Teofrasto, sin llegar a formular una doctrina neoplatónica de la sensación, se le aproxima en ciertos aspectos, y en todo caso más que Aristóteles. Esto es probablemente lo que ocurre con la noción de la asimilación (ó $\left.{ }^{\prime}{ }^{\prime} \omega \sigma r \varsigma\right)^{17}$, un concepto que Aristóteles había utilizado casualmente en el marco de su explicación del mecanismo de la sensación, pero que estaba lleno de dificultades, y en la cual Teofrasto se detuvo.

El análisis aristotélico de la sensación comienza en el capítulo 5 del segundo libro del De anima. Se lee en su desarrollo:

T4 = Aristóteles, Acerca del alma, II, 5, 418a4-6

[Lo que tiene la capacidad de sentir] padece en la medida en que no es semejante [sc. al objeto percibido], sino una vez que ha sido afectado, deviene semejante y es tal como éste ${ }^{18}$.

La frase sigue a una referencia de la tesis que ya había sido formulada con anterioridad, según la cual la relación de la sensación con lo sentido es una relación de potencia al acto:

principalmente las p. 45s. sobre Prisciano. En relación al aspecto que aquí nos concierne (la sensación como activación de una forma interior), los elementos esenciales ya se encuentran en Plotino, ver Emilsson 1988, en particular 133ss.

17 Ver infra, T7.

${ }^{18}$ El sujeto tò aíoĐitıkóv es tomado de la frase precedente (citada infra, nota 18). El término, que traduzco de manera neutra como «lo que tiene la capacidad de sentir», no designa necesariamente, de manera restrictiva el órgano sensorial (que Aristóteles frecuentemente

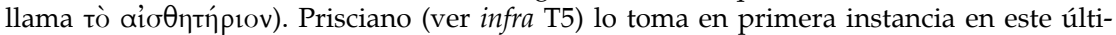
mo sentido, y es también el caso de la mayoría de los intérpretes (con la excepción de M. Burnyeat, quien guarda su sentido amplio). La continuación de T5 sugiere que Teofrasto mismo (si no es Prisiciano quien aquí habla) había distinguido dos casos posibles: «lo que tiene la capacidad de sentir» puede ser el alma, y no solamente los órganos. Es cierto que numerosos textos paralelos de Aristóteles se refieren al órgano (ver Sorabji 1991: 2012ss) y que es también el término que aparece bajo la pluma de Prisciano/Teofrasto (ver T5, T8 y T9, donde se notará, sin embargo, la mención «centro» sensitivo, tò kúprov). 
«lo que tiene la capacidad de sentir es en potencia así como lo sentido es por su parte en acto, como se ha dicho» ${ }^{19}$. Esta tesis orienta de entrada la interpretación del término «asimilación». Pero Teofrasto consideró que éste se podía prestar a confusión: la palabra, dice, es «rara» (atopon). El comentario de Prisciano que comienza tras una frase de transición ${ }^{20}$, da cuenta de la explicación que Teofrasto había dado de esta dificultad:

T5 = Prisciano, p. 1, 3-8 (273FHS\&G)

Puesto que Aristóteles quiere que los órganos sensoriales, cuando son cambiados por los sensibles, se asimilen integralmente a los sensibles por el hecho de padecer, <Teofrasto> se pregunta en qué consiste esta asimilación. Pues al tratar de los órganos sensoriales, y aun más del alma, parece extraño que la asimilación integral al color, a los sabores, al sonido y a la forma, sea posible. Pues bien, él mismo dice que la asimilación tiene lugar en conformidad con las formas (eidê) y las articulaciones formales (lógoi), sin la materia ${ }^{21}$.

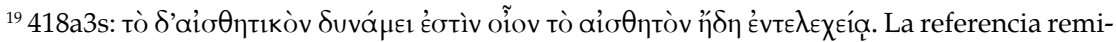
te a 417a12 (distinción general entre los dos sentidos del término «sensación», en potencia y en acto), pero no es sino al momento de hacer la referencia que Aristóteles precisa la relación entre ambos, distinguiendo al interior de la sensación «lo que tiene la capacidad de sentir» de «lo sentido».

${ }^{20}$ En la tradición manuscrita, el tratado comienza de manera abrupta: «Su siguiente ob-

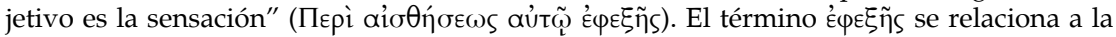
disposición de los temas en el libro de Teofrasto, tan sólo designado por la palabra aủtw̃, pero muestra que la metáfrasis ya había tratado otros temas diferentes al de la sensación, correspondiendo muy probablemente al contenido del libro I del tratado Acerca del alma — tanto del de Teofrasto como el de Aristóteles (ver supra, n. 9).

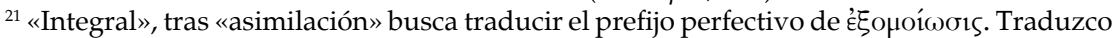

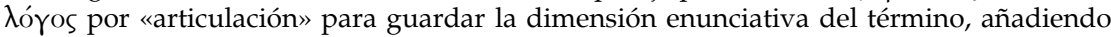
«formal», lo que tiene la doble ventaja de justificar la relación, de hecho perfectamente aristotélica, entre عĩos y $\lambda$ ó $\gamma$ os y de subrayar la continuidad entre el logos aristotélico y el logos de los neoplatónicos (el que I. y P. Hadot traducen por «razón formal»; ver Hadot 1997: 43).
} 
Esta última frase, ya sea que el sujeto sea Teofrasto o Aristóteles, remite a una fórmula célebre del De anima II, 12, 424a17$24^{22}$. La dificultad a la cual se enfrenta Teofrasto es la siguiente: si el sensible es el color (para la vista), el sabor (para el gusto), el sonido (para el oído), e incluso la forma (un ejemplo de sensible común), ¿qué puede significar esta asimilación de lo sentido por lo sensible, sino que el órgano sensorial se colora, deviene sonoro, etcétera? ${ }^{23}$ La respuesta de Teofrasto consiste en descartar esta interpretación material de la asimilación, y Teofrasto duplica esta dificultad distinguiendo explícitamente el caso del órgano sensorial del caso del alma: aun cuando se admitiera que el órgano corporal sufre una asimilación de este tipo, no podría suceder lo mismo con el órgano que propiamente hablando percibe y que con mayor razón posee «la capacidad de sentir».

La solución de Teofrasto consiste en leer la frase problemática a la luz del famoso pasaje del capítulo II, 12, 424a1724 que, si bien no hace uso explícito del término «asimilación», muestra que la teoría aristotélica de la sensación busca construirse independientemente de la referencia a la materia de lo sensible ${ }^{24}$ :

T6 = Aristóteles, Acerca del alma, II, 12, 424a17-24

De manera general, hay que comprender respecto a toda sensación que la sensación es lo que tiene la capacidad de recibir las

\footnotetext{
${ }^{22}$ La fórmula figura en Acerca del alma II, 12, 424a17-24 (ver infra, T6).

${ }^{23}$ Ver Ross 1967 (1961): 25: «This description of sense-perception as a power of assimilation to the sensible objects is, it is hardly to be said, an oversimplification of the matter; it would imply, if taken strictly, that in seeing a green object the eye actually becomes green». Es la interpretación defendida por Sorabji. Ver supra n.1 y 2.

${ }^{24}$ En los comentaristas modernos, los partidarios de una interpretación no fisiológica del proceso sensorial aducen evidentemente a este pasaje: ver Burnyeat 1996: 163 (contra Sorabji 1992: 213ss).
} 
formas sensibles sin la materia, como la cera recibe la huella del anillo sin el hierro o el oro, y recibe la huella del oro o del bronce, pero no en tanto que es del oro o del bronce. De igual manera, la sensación de cada cosa [sensible] padece bajo el efecto de la cosa que posee el color, el sabor o el sonido, no de la manera como cada una de estas cosas se dice, sino en tanto que posee tal cualidad y en conformidad con su articulación formal $(\log o s)$.

El objetivo de esta referencia es claro: si la sensación recibe sólo la «forma» (o aún más el logos) ${ }^{25}$ del sensible, la asimilación entre la sensación y su objeto de la cual Aristóteles habla en el capítulo 5 no puede ser sino de naturaleza puramente formal. No ocurre entonces que el órgano sensorial (o el alma, si de ésta se trata) sufra cierta coloración; lo que la sensación deviene es la forma del objeto sensible.

Tal exégesis parece estar en conformidad con la intención de Aristóteles, donde el uso de la palabra «asimilación» está subordinado a la distinción entre la potencia y el acto. Tiene en todo caso la capacidad de satisfacer a Prisciano, pues constituye un primer paso en el rechazo de toda explicación que haga de la sensación una afección del alma. Es cierto que desde el punto de vista de Prisciano, ni Aristóteles ni Teofrasto van suficientemente lejos en este sentido. Para un neoplatónico, la sensación es, propiamente hablando, una actividad del alma, si es cierto que la forma, lejos de venirnos de afuera, reside previamente «en nosotros» ${ }^{26}$. Ahora bien, Aristóteles y Teofrasto, sí desmaterializan, no desexternalizan:

\footnotetext{
${ }^{25}$ Aún cuando se pueda adjudicar la equivalencia a Prisciano, ésta remonta verosímilmente al propio Teofrasto.

${ }^{26}$ La doctrina es constante desde Plotino (ver Emilsson 1988: 133ss). Hadot 1997, cita y comenta la Sentencia 16 de Porfirio (ver 7, 3 y 8, 5, Lambetz).
} 
T7 = Prisciano, p. 3, 27-31 (273 FHS\&G)

Cuando, entonces, Teofrasto quiere que la asimilación tenga lugar en conformidad con las formas y la articulación formal (logos) sin la materia, lo aceptamos: pero no es que éstas se introduzcan simplemente del exterior; por el contrario, surgiendo en el seno de la vida, de acuerdo a la actividad sensitiva, a partir de las articulaciones formales internas (apo tôn endon logôn), tienden hacia los $<$ objetos $>$ externos y se asimilan a ellos ${ }^{27}$.

Las vías de Prisciano y de Teofrasto se separan aquí. Sin embargo, la paráfrasis de Prisciano contiene indicaciones bastante precisas e interesantes sobre la manera en que Teofrasto había concebido el proceso físico de la asimilación formal.

T8 = Prisciano, p. 15, 2516 (277B, 11-24 FHS\&G)

Él [Teofrasto] prosigue diciendo que, para ciertos sentidos, el órgano sensorial parece ser del mismo género que los sensibles. En efecto, la lengua percibe los sabores por medio de lo húmedo, y el oído la voz por medio del aire circundante que se mueve. Se pregunta entonces por qué no sucede también lo mismo en los otros casos, y cómo lo semejante padece bajo el efecto de lo semejante, el aire bajo el efecto del aire exterior y lo húmedo bajo el efecto de lo húmedo. ¿Acaso esta frase no es en efecto semejante para los otros casos? Para cada sentido, en efecto, el órgano sensorial es tal que el substrato exterior de la actividad de los sensibles - lo transparente para la vista, lo transaudible para el oído (pues no es en tanto que aire sino en tanto que transaudible que el oído recibe el sonido) y lo transoliente para el olfato. Es por ello que aquí también lo húmedo o el aire son intermediarios. Lo húmedo es el

${ }^{27}$ El pasaje se sitúa al término del desarrollo introducido por T5. 
intermediario en el caso del gusto y, en el caso del tacto, lo que tiene la capacidad de recibir tales cualidades táctiles. Y no es lo semejante lo que padece bajo el efecto de lo semejante, sino lo que es en potencia bajo el efecto de lo que es en acto: ciertamente no como el aire bajo el efecto del aire, sino como lo transaudible que está en potencia, bajo el efecto de lo transonoro que está en acto.

Teofrasto comienza resaltando dos dificultades a propósito de la «similitud» supuesta entre el órgano y su objeto: a) ésta no es aparente sino en el caso del gusto y de la vista, ¿qué sucede entonces con los otros sentidos?28; b) admitiendo que la similitud se confirmase para todos los sentidos, ¿cómo explicar que el mecanismo de la sensación dé lugar a una «afección», si es verdadero que, como Aristóteles sostiene, lo semejante no padece bajo el efecto de lo semejante? ${ }^{29}$ La respuesta a estas dos preguntas, típicamente introducidas por $\dddot{\eta}$, consiste en sostener sucesivamente: a') que a pesar de las apariencias, la similitud buscada está presente en cada uno de los cinco sentidos, aun cuando no se encuentre en donde lo supone el enunciado de la dificultad; b') que el principio aristotélico de la impasibilidad de lo semejante no es violentado, si bien es cierto que la similitud en cuestión —así como la afección- debe tomarse en un sentido especial del término «similitud $»^{30}$.

\footnotetext{
${ }^{28}$ El pasaje sugiere que Teofrasto, si bien había descartado la interpretación material de la asimilación, había también citado, en el contexto de la discusión intermedia, argumentos que parecían ir en su favor, como la homogeneidad material de ciertos órganos con su sensible.

${ }^{29}$ El principio es enunciado en particular en Acerca de la generación y la corrupción, I, 7, 323b18-20, en el curso de la discusión a la que remite Acerca del alma, II, 5, 416b35-417a2.

${ }^{30}$ Que el conjunto del pasaje, y en particular los neologismos «transaudible» $\mathrm{y}$ «transoliente», en la sección introducida mediante «¿Acaso la sentencia no es en efecto semejante...?», remonta a Teofrasto es confirmado por Filopón en su comentario al tratado Acerca del alma, ad 419a33 (ver p. 354, 12-16 Hayduck = 277C FHS\&G): «Aristóteles dice que el poder co-
} 
Teofrasto procede entonces a profundizar el sentido y las condiciones de posibilidad de la «asimilación». Dos puntos deben en este momento señalarse:

1. La similitud formal que supone el proceso sensorial ${ }^{31}$ se sitúa en menor medida entre el órgano sensorial y el sensible que entre el órgano sensorial y una entidad que Teofrasto designa en primera instancia mediante una expresión perifrástica: «el subs-

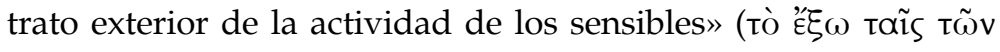

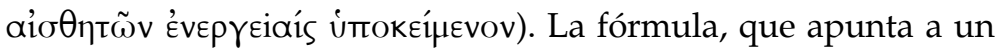
mayor grado de precisión, designa de hecho el «intermediario», como lo indican los ejemplos inmediatamente citados a manera de ilustración.

2. Teofrasto introduce dos innovaciones terminológicas. De entrada, al forjar una denominación especial para dos de los intermediarios que — dejando a un lado el «transparente»— son anónimos en Aristóteles. Sobre el modelo lingüístico del «transparente», Teofrasto

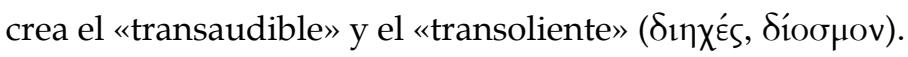

3.

Esta primera innovación va acompañada, a propósito del sonido, de una distinción entre el «transaudible» ( $\delta_{\imath \eta} \chi \chi^{\varepsilon} \varsigma$ ) y el

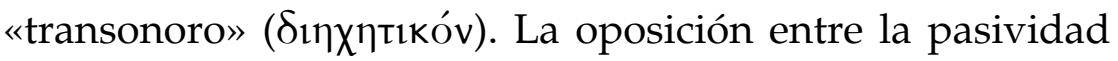

mún al aire y al agua mediante el cual los olores son transportados son anónimos: pero quienes vinieron después, entre los cuales se encuentra Teofrasto, le dieron el nombre de "transoliente", así como al que transporta el sonido le dieron el nombre de transaudible». Baltussen $(2000 ; 248$ s.) pone en duda la paternidad de Teofrasto, pero sin convencer. El uso que los comentadores posteriores hacen de estos términos simplemente testimonia del éxito de la terminología (por diosmos, ver Alejandro, comentario al De sensu de Aristóteles (p. 89, 2), donde el término «algún», $\tau \iota \varsigma$, apunta sin duda a Teofrasto). Al parecer Teofrasto habría renunciado al empleo de «transgustativo» y de «transtáctil», a los que respectivamente nombra «húmedo», $\mathrm{y}$, aún más prudentemente, «lo que tiene la capacidad de recibir las cualidades táctiles».

${ }^{31}$ La distinción entre homogeneidad material y similitud formal podría explicar que Teo-

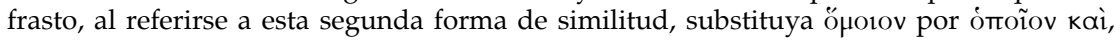
probablemente muy vinculada a la primera. 
del intermediario y la actividad de lo que éste recibe, que

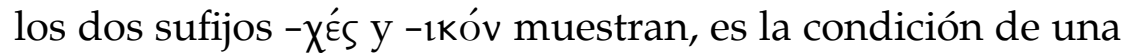
asimilación por así decir prefigurada por la identidad misma de los radicales. De esta manera, la afección es recibida, por parte del receptor, por el «transaudible», que es en potencia, bajo el efecto del «transonoro», que, del lado del objeto, es en acto. Aun cuando no contemos sobre este punto con información relativa a los otros sentidos, es claro que Teofrasto debía, al menos en principio, admitir la exigencia de una división análoga para cada uno de ellos: la homogeneidad estructural de las diferentes especies de sensación lo exige. Inspirándonos en el mismo esquema lingüístico, podemos llamar «mediable» y «mediante» los dos aspectos aislados por Teofrasto. Lo que este dúo permite precisar es la naturaleza de la similitud genérica (representada por el radical) y la naturaleza de la diferencia específica (representada por el sufijo). El lenguaje de la «asimilación» está entonces justificado, a pesar de que lo que está en juego es la actualización de una potencia.

La doble innovación terminológica de Teofrasto permite mostrar cómo el uso de un término aparentemente impropio («asimilación») responde de hecho a la estructura profunda de la teoría aristotélica. En esta medida, constituye un esfuerzo de sistematización respecto al cual contamos con otros ejemplos en la obra del discípulo. En este caso, la sistematización va acompañada de cierta inflexión. Los dos términos que Aristóteles subsume bajo el dúo potencia/acto (dunamis/ energeia) son, por una parte, la sensación (aisthêsis), y lo sentido (aisthêton), por la otra. Teofrasto, sin decir más, llama la atención sobre el elemento intermedio, identificando el correlato propiamente hablando de la sensación al «mediante» (en el caso del oído, el «transonoro»). 
Un nuevo afinamiento conceptual, cuya formulación es conservada por Prisciano en un largo pasaje relativo al sonido y el mecanismo de la audición, permite comprender mejor el papel asignado por Teofrasto a este «mediante», y la relación que mantiene con el sensible.

T9 = Prisciano, p. 17, 7-24 (277B, 50-68 FHS\&G)

Pero si el sonido proviene del exterior hasta el oído, ¿como puede él [sc. Aristóteles] afirmar que ninguna sensación se produce cuando el sensible está en contacto? Pues en el caso del olfato, igualmente, inhalamos el olor por la respiración hasta que alcanza, evidentemente, el centro [sensitivo].

¿No será que el sensible se sitúa donde se da el encuentro, donde se sitúa el buen olor y donde se sitúa el color? Ninguno entre ellos puede separarse de los órganos sensoriales, si debe haber sensación. Pero la forma de la actividad proveniente de ésta, que penetra en el intermediario, debe también estar presente en el órgano. Pues es evidente, como él mismo lo propone [sc. Teofrasto], que una sensación no padecerá ningún cambio si nada viene hasta ella proveniente de lo sensible. Y de hecho, el color cambia la vista gracias a lo transparente, el intermediario actuando y colaborando también por una parte, quien igualmente padece de alguna manera un cambio bajo el efecto de lo visible —de qué manera, ello ha sido ya explicado en la medida de lo posible--, y la sensación no es [sensación] del intermediario, sino más bien del agente. En efecto, no es lo mismo la acción (poiêsis) y el agente (poioun), ni tampoco la actividad (energeia) y el actuante (energoun). Ahora bien, el agente actúa conforme a la acción, y el paciente padece bajo el efecto del agente, pero en conformidad con la acción que de él proviene y no bajo el efecto de la acción. Tan es así que lo que sentimos no es la actividad proveniente del sensible, sino el sensible en conformidad con la actividad que de él proviene; y 
entonces no es el intermediario, sino la forma de la actividad del emisor emitida en el intermediario.

La estructura del pasaje es idéntica a la del texto precedente (T8), donde se abre enunciando una dificultad (que en esta ocasión trata de la doctrina de Aristóteles) seguida de una solución elaborada («Acaso...» = «¿No será que...?») ${ }^{32}$.

Teofrasto comenta aquí Acerca del alma, II, 8. Compara la definición aristotélica de lo sonoro como «lo que tiene la capacidad de mover un aire continuamente hasta el oído» (420a3-19) con la teoría del intermediario que parece contradecir. Esta última implica en efecto que la sensación se efectúa mediatamente, es decir, sin contacto directo entre el sensible y el órgano, como Aristóteles mismo afirma en el capítulo precedente (ver 419a25-31, y 423b20-25). Ahora bien, admitir que el sonido es transmitido sin solución de continuidad del aire externo al aire interno, parece implicar la existencia de dicho contacto ${ }^{33}$.

Teofrasto no está a la búsqueda de una respuesta ad hoc. Lo que indica sobre la audición -y del olor, que le está inmediatamente asociado- es interesante, pues ilustra, siempre en conformidad con la tesis de la homogeneidad estructural de todas las sensaciones, una dificultad general. Es lo que indica el desarrollo de la solución, que no sólo menciona el golpe del sonido ( $\pi \lambda \eta \gamma \eta ́)$ y del olor ( $\left.\alpha{ }^{\prime} \rho \mu \alpha\right)$, sino también del color $(\chi \rho \tilde{\omega} \mu \alpha)$.

\footnotetext{
32 La objeción y su respuesta forman parte, a propósito de este capítulo, de una serie construida sobre el mismo modelo.

${ }^{33}$ La expresión aristotélica évòs å épos ouvexéía, 420a3, que pone el acento sobre la unicidad del aire involucrado en el mecanismo de la audición es particularmente de llamar la atención.
} 
Teofrasto admite explícitamente la necesidad de que «algo» vaya del sensible al órgano si debe darse cambio sensorial ${ }^{34}$. Pero ese algo no es el sensible mismo, el que permanece situado

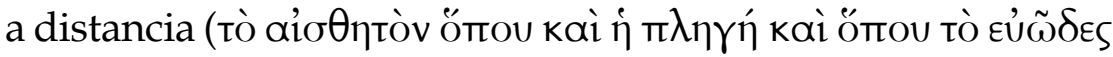

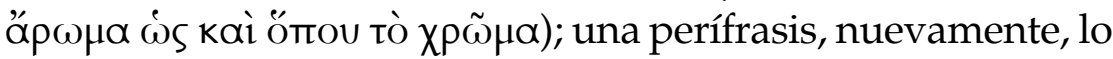
designa como «la forma de la actividad proveniente de éste,

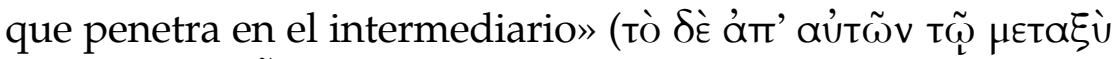

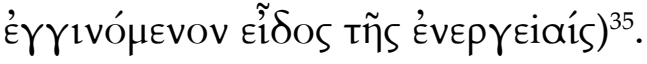

Para comprender lo que implica exactamente esta «forma de la actividad», hay que regresar a la distinción, establecida en la continuación del desarrollo, entre (a) «la actividad

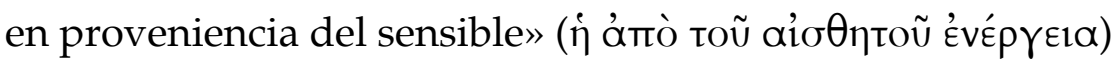
y (b) «el sensible, pero de acuerdo a la actividad que provie-

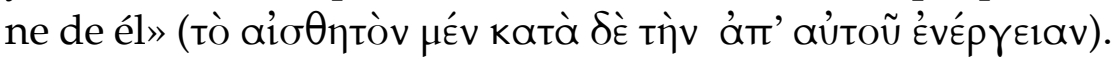
Teofrasto niega que la actividad (a) pueda ser el objeto de la sensación, pero afirma que sí es el caso del sensible considerado desde un cierto punto de vista (b) ${ }^{36}$. La razón es que la actividad, en cuanto tal, no forma parte de las entidades que pueden ser percibidas. «El sensible considerado en su actividad», por su lado, satisface las dos condiciones que, por difíciles de conciliar que puedan parecer, deben ser mantenidas simultáneamente. En la medida en que el sensible es percibido, pero solamente de acuerdo a la actividad que le es propia, no hay contacto directo entre el órgano y el sensible

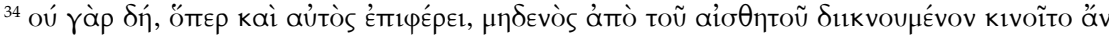

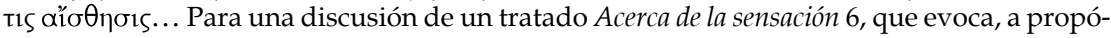
sito del sonido y del olor, el desplazamiento progresivo de los movimientos provenientes de los sensibles (446a21ss), ver Johansen 1998: 140-143.

${ }^{35}$ Encontramos aquí el término Ễosos, del cual vimos la función en T4, integrado a una

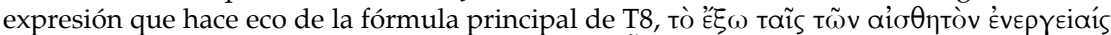

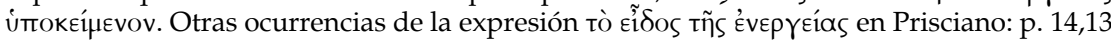
y 32. En ambos casos se opone a rá $\theta$ os.

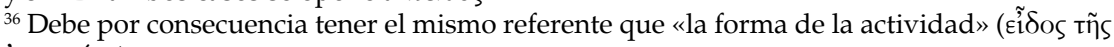
ÈvepYéías).
} 
mismo, que permanece a distancia; $y$, sin embargo, lo que la percepción percibe es efectivamente la fuente de esta actividad, es decir, el objeto mismo. La «forma de la actividad proveniente de los sensibles» se hace así cargo de la difícil tarea de asignar un papel al intermedio sin amenazar la objetividad de la relación.

Esta explicación remite, sin embargo, a una última distinción entre el agente (to poioun) y su acción (hê poiêsis), el actuante (to energoun) y su actividad (hê energeia). El sentido, y el beneficio de esta doble distinción es excluir los sustantivos que designan la acción misma (poiêsis, energeia) de la clase, de lo que tiene la capacidad de actuar y entonces de ser agente, lo que quiere decir, desde un punto de vista gramatical, de ser complemento del agente. Sólo pueden ser introducidos por la preposición úró nombres de agentes tales como tò пo1oũv (el agente) o tò éveproũv (el actuante). Los sustantivos de acción, por su parte, están regidos por la preposición katá. Es entonces un grave error gramatical el que cometeríamos si pensáramos que podemos «percibir la actividad». No lo podemos hacer por la simple razón de que la actividad no actúa. Inversamente, lo que el intermediario transmite no es el agente, sino sólo la forma de su actividad y ello sin solución de continuidad.

Si Prisciano conserva el aparato conceptual que Teofrasto había elaborado para dar cuenta de la asimilación formal entre el objeto sentido (es decir, «la forma de la actividad del sensible») y la potencia de esta forma que es la sensación, no da, en cambio, todos los elementos para comprender el mecanismo de esta transmisión formal, como lo que podríamos llamar la física, o posiblemente mejor la cuasi-física. Sin embargo, es probable que Teofrasto se haya empeñado con dicha explicación puesto que, en el pasaje mismo que Pris- 
ciano reproduce o calca, se hace referencia a un pasaje anterior del tratado de Teofrasto que explicaba «en la medida de lo posible» la manera en que el intermediario era alterado, y esta alteración transmitida hasta el órgano sensorial (cf. «de qué manera, ello ha sido ya explicitado en la medida de lo posible»). Prisciano se contenta con hacer notar que la alteración del intermediario bajo el efecto de lo visible (el color) se efectúa «de cierta manera». Es muy probable que el adverbio $\pi \omega \varsigma$ no apunte aquí al empleo impropio de un término ( $\pi \omega_{\varsigma}$ alienans), en este caso el que consistiría en hablar de «alteración» en relación a la sensación. De hecho, tenemos la buena suerte de que Simplicio haya conservado en su comentario al De anima algunos indicios sobre la manera en la que Teofrasto había buscado comprender más precisamente el proceso:

T10 = Simplicius, In De anima, II, 7, 419a13, p. 136,20-29 Hayduck (279FHS\&G)

El transparente es cambiado por el color en tanto que recibe la actividad que proviene de él, y cambia el órgano sensorial en tanto que transmite esta misma actividad, y no en tanto que actúa y cambia la vista por él mismo, independientemente del color —como el hierro caliente, aun cuando el fuego que calienta ya no esté presente- sino en tanto que transmite la actividad del color, como la pala, que ella misma movida por la mano, mueve la piedra. Es la razón por la cual lo transparente, cuando se ha abstraído el color, no mueve más la vista en conformidad con él. Y si lo transparente moviese por sí mismo la vista, sería necesario que fuese, él, objeto de la sensación, y no lo colorado. Pero de hecho, es éste último lo que percibimos, así como la distancia intermedia. Expliqué esto de manera más clara en el compendio de la Física de Teofrasto.

Apoyándose en Teofrasto, Simplicio comenta en este momento la afirmación de Aristóteles según la cual «el color 
cambia lo transparente y es por éste último, que es continuo, que el órgano cambia» ${ }^{37}$. Simplicio no hace aquí sino resumir un desarrollo que había consagrado a la cuestión en otro lado (la obra por supuesto se ha perdido) ${ }^{38}$. Lo que nos proporciona es, sin duda alguna, nada menos que un resumen de la explicación a la que Teofrasto remite en el pasaje correspondiente conservado por Prisciano, cuando dice haber explicado ya el tema con anterioridad (T9). Lo que aquí se analiza es, en efecto, la manera en la que se deben concebir los dos momentos extremos del proceso visual, a saber: 1) la alteración de lo transparente por el color y 2) la alteración del órgano por lo transparente:

1. De lo transparente se dice recibir ( $\mu \varepsilon \tau \alpha \lambda \alpha \mu \beta \beta a ́ v o v)$ y transmitir

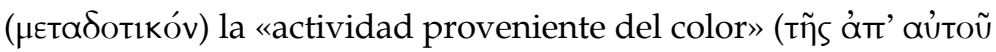

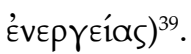

2. Una distinción es introducida entre dos tipos de comunicación de la actividad: a) una forma de delegación, o de depósito, que no requiere de la presencia del agente para perdurar como en el caso del hierro, el cual conserva el calor aún cuando se aleje su fuente; b) una comunicación pensada sobre el modelo de la transmisión continua, que Teofrasto describe recurriendo a un verbo que parece haberse vuelto técnico, $\delta 1 \alpha \beta ı \beta a ́ \zeta \varepsilon ı v ~(« f r a n q u e a r »)^{40}$.

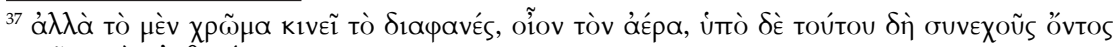
kiveĩta tò aiơntínpiov, 419a13ss.

${ }^{38}$ Se trata del compendio de la Física de Teofrasto. Por otro lado, a esta obra sólo se le constata en un pasaje de Simplicio en su comentario al tratado Acerca del alma (p. 136, 29 Hayduck), si es efectivamente el autor, y no Prisciano mismo, siguiendo la tesis de F. Boissier y C. Steel (discusión crítica de esta opinión en Hadot 1978; 163ss, quien mantiene firmemente su atribución a Simplicio en Hadot 1990: 291ss). Steinmetz (1964: p. 110, n. 4), corrige <'́v toĩs> Ė's тìv Émıtopì̀v... (=en el comentario que Simplicio habría escrito de un compendio que Teofrasto habría hecho de su propia Física, ver supra, n. 6)

${ }^{39}$ Ver T9. Se notará, sin embargo, que Simplicio no respeta la distinción entre la «actividad» y la «forma de la actividad».

${ }^{40}$ Prisciano, por su cuenta, también utiliza el término (p. 12, 30 y 14, 20).
} 
Es evidentemente a esta última categoría que pertenece la visión. Este modelo está relacionado con el problema de saber cómo es posible que lo que percibimos sea la cosa misma, y no el intermediario; un punto que es explícitamente mencionado por Simplicio ${ }^{41}$. Para nosotros, la respuesta se resume en una analogía. Para ilustrar la transmisión cinética de «la forma de la actividad», Teofrasto había invocado las propiedades de la pala (ó pox入ós). Así como la mano -gracias a la pala- mueve la piedra sin tocarla directamente, pero conservando entre ambas una innegable continuidad, lo visible (y de manera general lo sensible) altera el órgano por mediación del medio, pero sin que el contacto sea interrumpido ${ }^{42}$.

Hubiéramos querido saber más, pero a partir de los elementos que hemos podido reunir del comentario de Teofrasto sobre la teoría aristotélica de la sensación, queda por lo menos una cosa clara con respecto al debate sobre la concepción aristotélica de la percepción sensible: si hubo «desmaterialización» de la doctrina en la tradición exegética, hemos de remontarla hasta este lector privilegiado de Aristóteles que fue Teofrasto. Si, por el contrario, se sostiene que la doctrina de Aristóteles ya está por sí misma desmaterializada (y el carácter de la exégesis de Teofrasto me parece hablar en este caso a favor de ésta segunda opción, es decir, la interpretación de Burnyeat), Teofrasto nos permite delimitar mejor

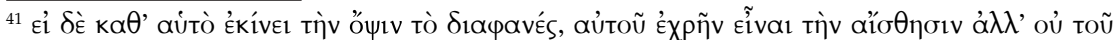

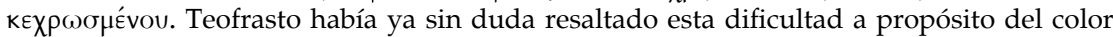
y de la vista. Es en todo caso evocada por Prisciano (p. 12ss): à $\lambda \lambda$ ' oút

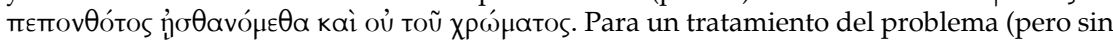
referencia a los textos aquí estudiados), ver Johansen 1998, capítulo 2, principalmente pp. $118-120$ y 135.

${ }^{42}$ Lo que generalmente se destaca del ejemplo de la pala es menos su capacidad para transmitir la fuerza, que su potencia para desmultiplicarla (es, por ejemplo, el caso al inicio de las Cuestiones mecánicas pseudoaristotélicas), pero no es este aspecto el que Teofrasto retiene en el marco de una teoría de la sensación. 
los contornos y las implicaciones de una física aristotélica de la sensación, una física que, en virtud del carácter cuasi-metafórico de su objeto (un «cuasi-cambio»), ameritaría probablemente la denominación de cuasi-física.

\section{Textos en griego}

T1 : Temistio, In De anima, III, 5, p. 108. 8-11 Heinze=307A17-21 FHS\&G

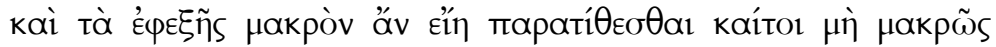

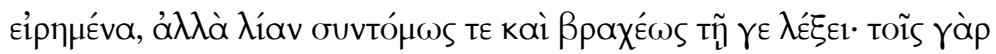

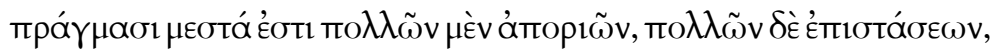

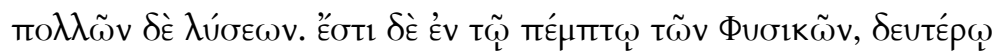

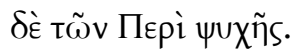

T2 : Prisciano, p. 22, 33s. = 297 FHS\&G

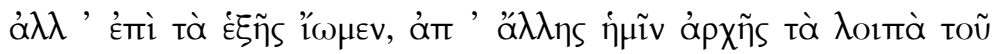

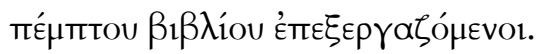

T3 : Prisciano, p. 7, 20-23 = 275A, 1-4 FHS\&G

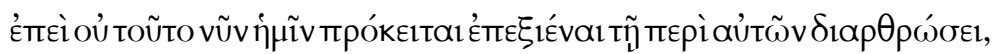

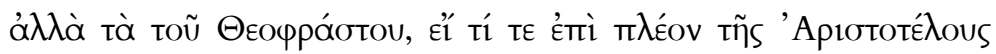

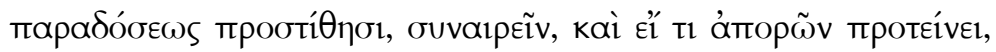

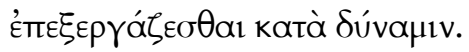

T4 : Aristóteles, De anima, II, 5, 418a 4-6

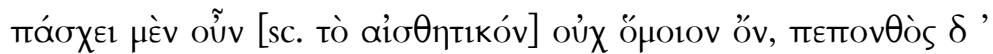

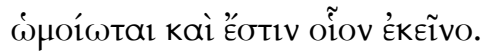

T5 : Prisciano, p. 1, 3-8 = 273, 1-7 FHS\&G

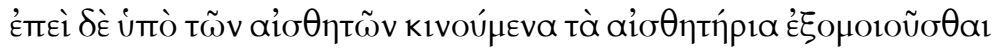

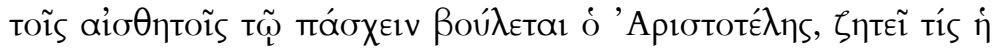

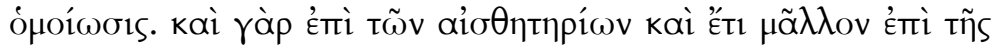

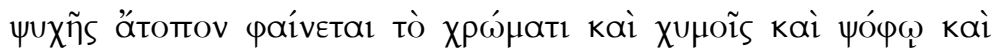




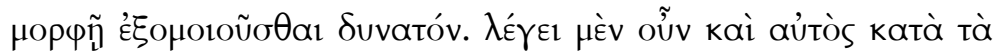

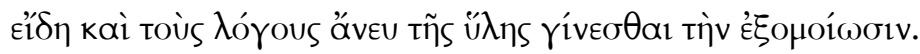

T6 : Aristóteles, De anima, II, 12, 424a 17-24

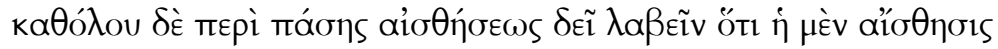

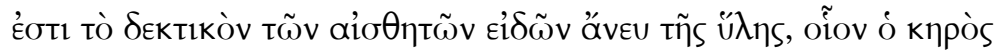

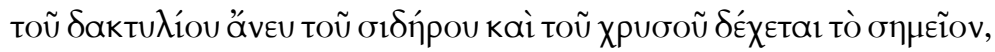

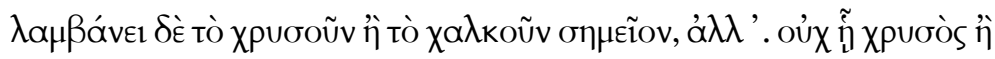

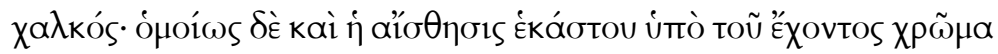

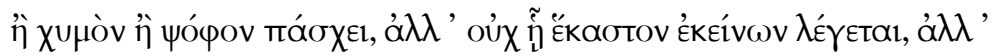

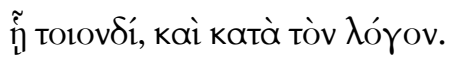

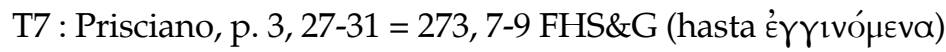

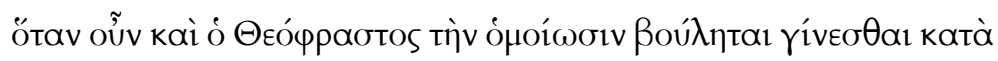

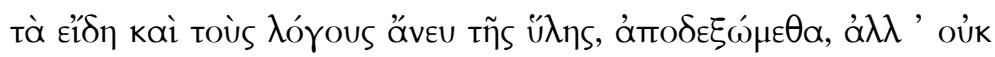

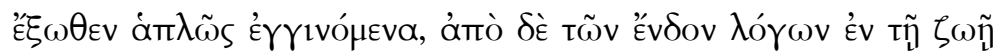

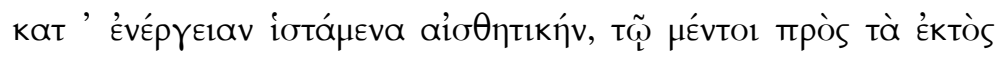

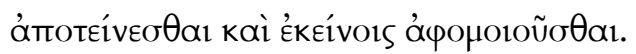

T8 : Prisciano, p. 15, 25 - 16, 6 = 277B, 11-24 FHS\&G

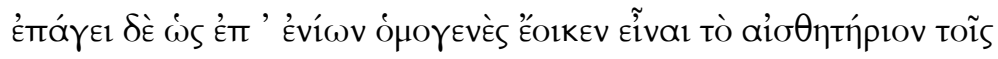
$\alpha i \sigma \theta \eta \tau$ ĩs.

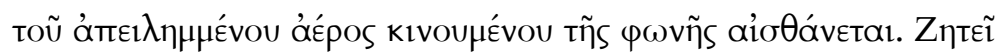

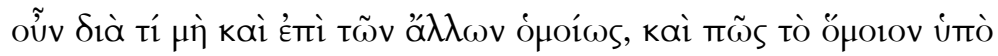
тои̃ ó

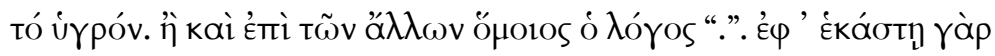

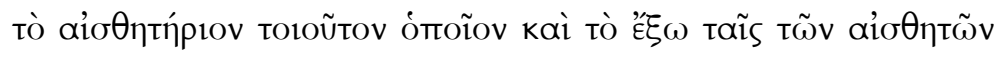

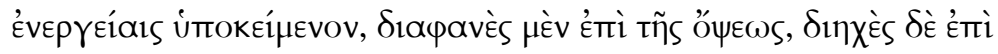

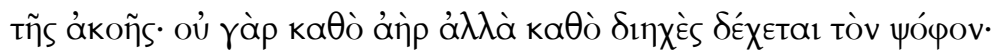

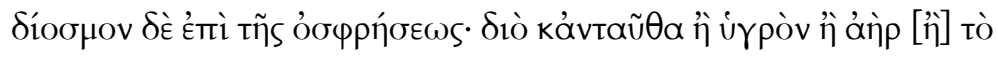

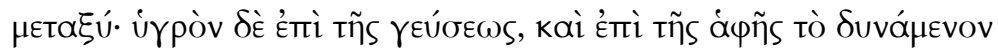

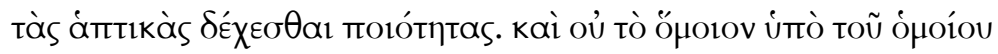

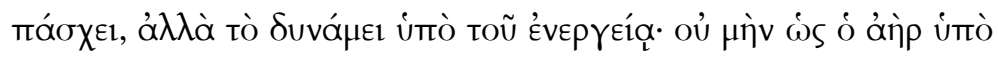

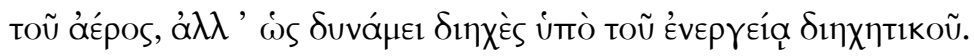


T9 : Prisciano, p. 17, 7-24 = 277B, 50-68 FHS\&G

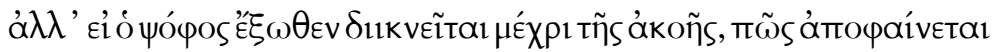

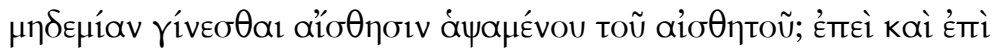

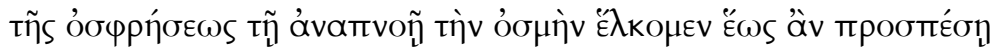

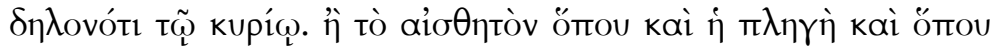

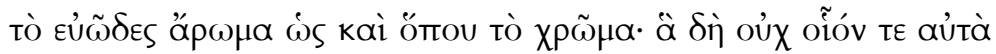

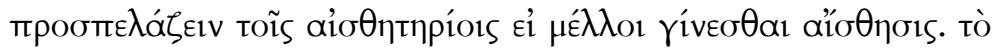

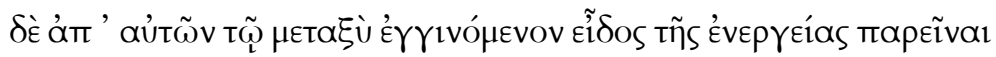

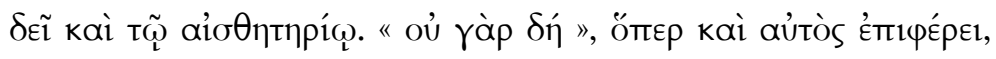

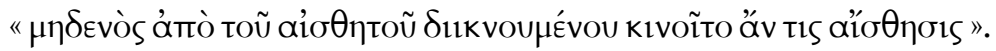

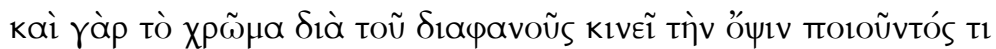

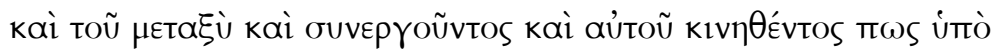

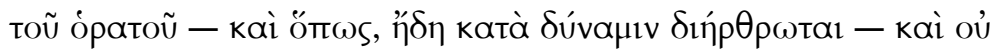

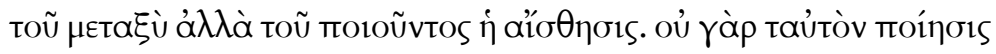

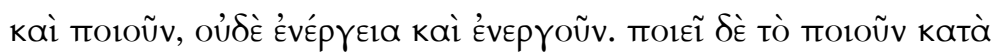

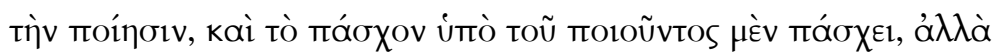

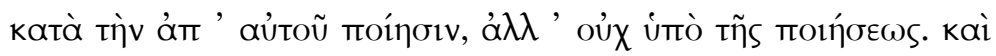

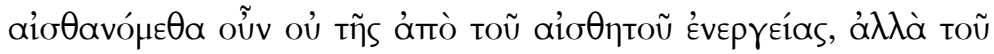

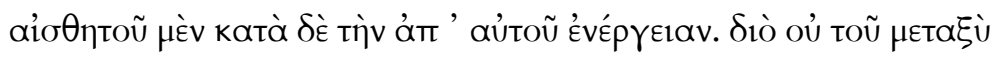

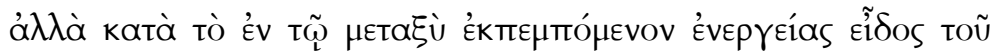

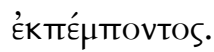

T10 : Simplicio, In De Anima II, 7, 419a13, p. 136, 20-29 Hayduck = 279 FHS\&G

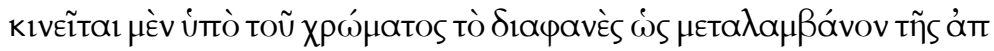

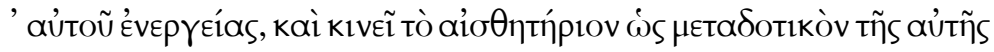

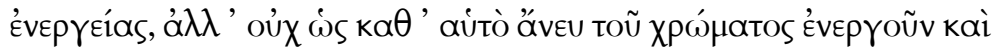

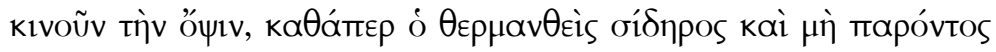

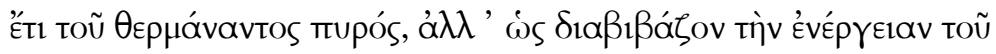

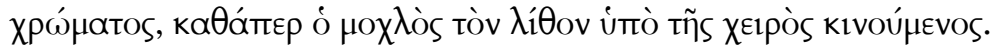

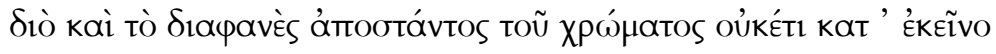

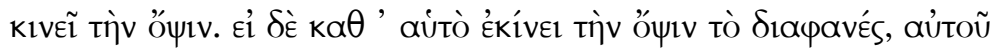

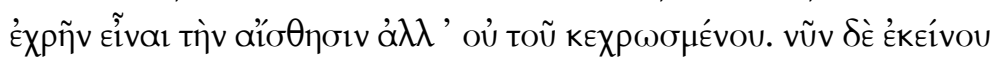




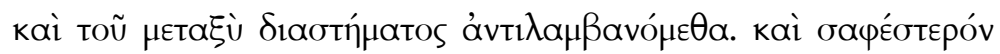

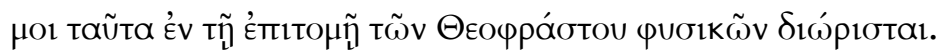

\section{BIBLIOGRAFÍA}

FHS\&G= Fortenbaugh, W.W., Huby, P., Sharples, R., Gutas, D. (1992). Theophrastus of Eresus. Sources for his life, writings, thought and influence, 2 vol. Leyde-New YorkCologne.

Burnyeat, M. F. (1996). «Aristote voit du rouge et entend un 'do': combien se passe-t-il de choses? Remarques sur De anima, II, 7-8 ». En: Romeyer Dherbey, G., Viano, C. (eds). Corps et âme, Sur le De anima d'Aristote (pp. 149167). Paris.

Burnyeat, M. F. (2001). «Aquinas on 'spiritual change' in perception». En: Perler, D. (ed). Ancient and Medieval theories of intentionality (pp. 129-153). Leiden-Boston-Köln.

Burnyeat, M. F. (2002). «De anima II 5». Phronesis 47; 28-90.

Emilsson, E. K. (1988). Plotinus on Sense-Perception. A Philosophical Study, Cambridge.

Gottschalk, H. B. (1967). Reseña de Steinmetz 1964. Gnomon $39 ; 17-26$.

Hadot, I. (1997). «Aspects de la théorie de la perception chez les néoplatoniciens: sensation, sensation commune, sensibles communs et conscience de soi». Documenti e Studi sulla Tradizione filosofica medievale 8; 33-85.

Johansen, T. K. (1998). Aristotle on the Sense-Organs. Cambridge.

Laks, A. 2007 (1998). «Le début d'une Physique. Ordre, extension et nature des fragments 142-144A/B de Théophraste». En: Histoire, Doxographie, Vérité. Etudes sur 
Aristote, Théophraste, et la philosophie présocratique (cap. 6). Louvain-la-Neuve: Peeters.

Laks, A., Most, G. W. (1993). Théophraste. Métaphysique. Paris. Mansfeld, J. (1996). «Aristote et la structure du De sensibus de Théophraste». Phronesis $41 ; 158-188$.

Ross, W. D. 1967 (1961). Aristotle's De anima. Oxford.

Sorabji, R. (1991). «From Aristotle to Brentano: The development of the concept of intentionality». En: Blumenthal, H., Robinson, H. (eds). Aristotle and the later Tradition (=Oxford Studies in Ancient Philosophy, Supplementary vol.; pp. 227-259).

Sorabji, R. (1992). «Intentionality and Physiological Processes: Aristotle's Theory of sense perception». En: Rorty, A., Nussbaum, M. (eds). Essays in Aristotle's De anima (pp. 195-225). Oxford.

Sorabji, R. (2001). «Aristotle on sensory processes and intentionality: a reply to Myles Burnyeat». En: Perler, D. (ed). Ancient and Medieval theories of intentionality (pp. 49-61). Leiden, Boston, Köln.

Steinmetz, P. (1964). Die Physik des Theophrastos von Eresos. Bad Homburg-Berlin-Zurich. 


\section{RESUMEN}

El autor examina a detalle una serie de fragmentos de Teofrasto sobre la doctrina de la sensación en Aristóteles. El problema al que se enfrenta Teofrasto es el siguiente: ¿qué querrá decir que la sensación es la asimilación de lo sentido por lo sensible?, ¿se da dicha asimilación en el órgano sensorial? En otras palabras: ¿será que el color «afecta» a la vista? ¿O será que lo que se recibe en la sensación es la forma? La búsqueda de una respuesta a estas interrogantes hace pensar, como anota el autor, que el papel de Teofrasto es esencial en el debate -también contemporáneo- sobre la supuesta «desmaterialización» de la percepción sensible en Aristóteles.

Palabras clave: Aristóteles; Teofrasto; sensación; color; percepción.

\section{ABSTRACT}

The author examines in detail a series of fragments from Theophrastus on the doctrine of sensation of Aristotle. The problem Theophrastus faces is the following: what does it mean that sensation is the assimilation by the sense of that which is felt? Does this assimilation take place in the sensitive organ? In other words, does color «affect» sight? Or might it be that the form is what is received in sensation? As the author points out, looking for an answer to such questions makes us deem the role of Theophrastus as paramount for the still contemporary debate regarding the alleged «dematerialization» of the sensitive perception in Aristotle.

Key words: Aristotle; Theophrastus; sensation; color; perception. 